\title{
Cepas de Pleurotus pulmonarius con alta capacidad productiva seleccionadas de micelios dicarióticos
}

\section{Strains of Pleurotus pulmonarius with high productive capacity selected from dikaryotic mycelia}

\author{
Dulce Salmones, Gerardo Mata, Rigoberto Gaitán-Hernández, Carlos Ortega
}

Red de Manejo Biotecnológico de Recursos. Instituto de Ecología, A.C. Carretera antigua a Coatepec 351, El Haya, C.P. 91073 ,

Xalapa, Veracruz, México.

\section{RESUMEN}

Antecedentes: Pleurotus pulmonarius es uno de los hongos comestibles comercialmente más importantes en el mundo. En México, su producción se sustenta en pocas cepas comerciales, por lo que es de interés seleccionar germoplasma altamente productivo y de buena calidad, adecuado para su propagación bajo las condiciones ambientales regionales. Objetivo: Obtener y seleccionar cepas altamente productivas de $P$. pulmonarius a partir del cruzamiento interespécimen de parentales comerciales.

Métodos: Se aislaron cultivos monospóricos de tres cepas, seleccionándose los de más rápido crecimiento micelial para determinar los tipos de compatibilidad y realizar las cruzas interespécimen. Los nuevos dicariones se cultivaron en paja de cebada para cuantificar: Precocidad en formación de primordios, eficiencia biológica (EB), tasa de producción (TP) y tamaño de los basidiomas, comparativamente con sus parentales.

Resultados y conclusiones: Se evaluaron 48 nuevos dicariones. Las EB's fluctuaron de 7.3 a 147.5 \% y las TP's de 0.14 a $2.59 \%$. Trece cepas progenie superaron el $80 \%$ de EB y 16 cepas el $1.5 \%$ de TP. La mayoría de las fructificaciones presentaron píleos de 5 a $15 \mathrm{~cm} \varnothing$. Aunque la productividad de las cepas progenie fue variable, algunos dicariones presentaron características aceptables para su aprovechamiento comercial.

Palabras clave: cultivo de hongos, eficiencia biológica, entrecruzamientos miceliales, hongo ostra de verano, tasa de producción

\section{ABSTRACT}

Background: Pleurotus pulmonarius is one of the commercially important edible mushrooms in the world. In Mexico, the production of this species is supported on few commercial strains; thus, it is a priority to select highly productive and good quality germplasm, suitable for its propagation under regional environmental conditions.

Objective: To obtain and select highly productive $P$. pulmonarius strains by the interspecimen breeding of commercial parentals.

Methods: Monosporic cultures from three strains were isolated, selecting the fastest mycelial growth cultures to determine the mating types and carry out the interspecimen crossbreeding. The new dikaryons were cultivated on barley straw to quantify the earliness of primordia initiation, biological efficiency (BE), production rate (PR) and basidiomes size, comparatively with their parents.

Results and conclusions: Forty-eight new dikaryons were evaluated. BE's fluctuated between 7.3 to $147.5 \%$ and PR's from 0.14 to $2.59 \%$. Thirteen progeny strains overcome BE's of $80 \%$ and 16 strains reached PRs upper to $1.5 \%$. Mostly, fruiting bodies developed pileus of 5 to $15 \mathrm{~cm} \varnothing$. Although the productivities of progeny strains were variable, some dikaryons presented acceptable characteristics for their commercial exploitation.

Keywords: biological efficiency, genetic crosses, mushroom cultivation, Phoenix mushroom, production rate

\section{ARTICLE HISTORY}

Received 20 September 2019 / Accepted 01 July 2020

Published on line: 15 July 2020
CORRESPONDING AUTHOR

\u Gerardo Mata, gerardo.mata@inecol.mx

ORCID: 0000-0003-1646-7003 


\section{INTRODUCCIÓN}

En la actualidad, el cultivo de hongos comestibles y medicinales es una actividad importante en México, ya que se produce un alimento de gran demanda, con buen precio en el mercado y que genera miles de empleos. Esta actividad inició en los años 30's del siglo pasado por migrantes europeos a través del cultivo de champiñones, específicamente Agaricus bisporus (J.E. Lange) Imbach, y se consolidó en décadas posteriores (Martínez-Carrera et al., 2016). Su desarrollo se robusteció a partir de los 70's con la introducción de especies de Pleurotus, conocidas comercialmente como "oyster mushrooms" en Europa y América del Norte, por su morfología que asemejan ostras y en México tomaron el nombre de "setas", ya que los primeros cultivos provenían de España, donde así se denominan a las especies comestibles. Aunque con anterioridad a las especies silvestres de Pleurotus se les denominaban oreja blanca, oreja de palo, oreja de cazahuate, hongo de patancán u hongo de izote (Guzmán, 2000), el término de setas se popularizó rápidamente y se mantiene en el mercado nacional.

Las especies de Pleurotus presentan ventajas de crecimiento sobre otros hongos cultivados, debido a su eficiente capacidad enzimática de biodegradación de una gran variedad de materiales lignocelulósicos (Mata et al., 2017). Además, sus ciclos de cultivo son cortos y quizás lo más atractivo desde el punto de vista comercial, algunas cepas son altamente productivas de basidiomas con tamaño y morfología de interés al consumidor (Chang y Miles, 2004).

En México se comercializa mayoritariamente Pleurotus pulmonarius (Fr.) Quél., debido a las preferencias de consumo de 'variedades claras', aunque entre los cultivadores no existe completa certeza sobre la especie que cultivan, ya que frecuentemente se confunde con Pleurotus ostreatus (Jacq.) P. Kumm., debido a su alta similitud morfológica e incluso confundidas en la bibliografía (Guzmán, 2000; Pánek et al., 2019). En condiciones naturales, $P$. pulmonarius y $P$. ostreatus pueden coexistir, aunque la primera se reproduce más favorablemente en ambientes más cálidos, lo cual sugiere que las condiciones ambientales han favorecido una eventual especiación alopátrica (Lechner et al., 2005; Shnyreva y Shtaer, 2006). A nivel comercial, P. pulmonarius resulta de gran interés para algunas condiciones ambientales del país, por lo que es prioritario disponer de cepas con alta producción de basidiomas de tamaño y morfología adecuada para el mercado regional, de preferencia con baja producción de esporas y rendimiento estable bajo diferentes condiciones de cultivo en ciclos cortos (Mata et al., 2013).

A pesar del desarrollo que ha tenido esta agroindustria a nivel nacional, los avances logrados en programas de entrecruzamiento genético de cepas realizados en instituciones nacionales no han incidido significativamente en la disponibilidad y reproducción masiva de germoplasma adecuado para las condiciones ambientales regionales, por lo que la producción nacional continúa sustentada en un limitado número de cepas.

En el caso de Pleurotus, al presentar un patrón de sexualidad heterotálico tetrapolar (Shnyreva y Shtaer, 2006), es factible realizar cruzamientos de esporas entre los tipos de apareamiento compatibles. Este es un proceso complejo, azaroso y requiere partir de cepas parentales con capacidad de heredar a su descendencia características de interés al mercado, como la cantidad y el tamaño de basidiomas (Avin et al., 2016). En el presente estudio, se aislaron basidiosporas de cepas comerciales de Pleurotus pulmonarius, que se entrecruzaron para obtener los dicariones correspondientes, con el objetivo de determinar la variación en el crecimiento micelial, desarrollo de primordios, peso y tamaño de los basidiomas entre las cepas parentales y progenie, con la finalidad de seleccionar germoplasma con potencial comercial.

\section{MATERIALES Y MÉTODOS}

\section{Cepas}

Se utilizaron las siguientes cepas de P. pulmonarius como germoplasma parental: IE-115 (Fungi Perfecti, K200, EUA), IE-739 fue donada por un productor comercial nacional y la IE-717 (=LGAM P57) fue proporcionada por el Dr. G. Zervakis de la Universidad de Agricultura de Atenas (Grecia). Las primeras dos cepas son cultivadas comercialmente en México. Todo el material genético está depositado en el Cepario de Hongos del Instituto de Ecología (Xalapa, Mex.) (WDCM 782).

\section{Obtención de micelios monospóricos y cruzas}

Se colocaron basidiomas maduros de las cepas parentales cultivadas en paja de cebada sobre papel estéril, con la finalidad de obtener las esporadas. Los cultivos monospóricos se aislaron de diluciones de basidiospo- 
ras en agua destilada estéril a una concentración de $1 \mathrm{x}$ $10^{6}$ esporas $\mathrm{mL}^{-1}$ (Gaitán-Hernández y Salmones, 2008). Se dispersaron alícuotas de $0.5 \mathrm{~mL}$ de las suspensiones de esporas en cajas de Petri con $20 \mathrm{~mL}$ de medio de agar con papa y dextrosa (PDA, Bioxon) e incubadas en la oscuridad a $26^{\circ} \mathrm{C}$. Después de 3 a $4 \mathrm{~d}$ de incubación, las esporas que germinaron fueron transferidas a otra caja de Petri con PDA para favorecer su desarrollo y corroborar su estado monocariótico, mediante la ausencia de fíbulas. Al menos 20 cultivos monospóricos se aislaron de cada cepa parental.

Se eligieron 12 cultivos monospóricos al azar de cada cepa parental para obtener sus tipos de compatibilidad (Eger, 1978). Estos cultivos fueron dispuestos en pares entre ellos, sin cruzas recíprocas. Paralelamente, se prepararon cinco réplicas de los monospóricos de cada cepa, 36 en total, para estimar su crecimiento micelial en PDA a $26^{\circ} \mathrm{C}$. La cuantificación del crecimiento se basó en las mediciones de los diámetros miceliales desarrollados, a partir de dos líneas marcadas perpendicularmente una de otra, cada tercer día de incubación (Salmones y Durán Barradas, 2001). De acuerdo con los resultados del análisis estadístico de los valores obtenidos, se eligió el cultivo monospórico más rápido de cada tipo de compatibilidad, cuatro en total por cada parental, para realizar las cruzas interespécimen.

Se estimó el crecimiento micelial en PDA tanto a las cepas parentales como a las cruzas interespécimen obtenidas, de manera similar al procedimiento seguido para los cultivos monospóricos. Estos resultados fueron analizados estadísticamente mediante un ANOVA y prueba de medias de Tukey para determinar diferencias significativas entre los diámetros miceliales desarrollados, con apoyo del paquete estadístico Statistica 7.1 (StatSoft, 2005).

\section{Cultivo de las cepas en paja de cebada}

El inóculo se preparó con semillas de sorgo (Sorghum vulgare L.), ajustadas una humedad de ca $30 \%$, colocadas en bolsas de polietileno y esterilizadas a $121{ }^{\circ} \mathrm{C}$ durante 1 h (Guzmán et al., 2013). Las semillas fueron inoculadas con porciones de micelio de cada una de las tres cepas parentales y de las 48 cruzas. Las muestras se incubaron a $26^{\circ} \mathrm{C}$ en la oscuridad hasta el cubrimiento total de los granos por el micelio.

El sustrato utilizado para la producción de las fructificaciones fue paja de cebada (Hordeum vulgare L.), previamente procesada en una trilladora para obtener fragmentos de 5 a $8 \mathrm{~cm}$ de largo. El sustrato se hidrató y fermentó durante $5 \mathrm{~d}$, con volteos periódicos (al menos 2 veces) para favorecer un proceso aeróbico. Posteriormente, el sustrato fue colocado en un túnel de pasteurización y tratado térmicamente con vapor a $65{ }^{\circ} \mathrm{C}$ por 6 h. La siembra consistió en mezclar la paja de cebada (humedad del $68 \%$ ) con el inóculo de cada cepa a un porcentaje de inoculación del 5 \% (base húmeda), en bolsas de plástico de $40 \times 60 \mathrm{~cm}$. El peso final de las muestras fue de $4 \mathrm{~kg}$ con 10 réplicas por cepa. Las muestras fueron incubadas a $26 \pm 1{ }^{\circ} \mathrm{C}$ en la oscuridad hasta el cubrimiento total del sustrato por el micelio. Después se colocaron en un área con iluminación (12 h luz/12 h oscuridad), concentración de $\mathrm{CO}_{2}$ $<1200$ ppm, humedad de $85 \pm 5 \%$ y temperatura de $26 \pm 3{ }^{\circ} \mathrm{C}$.

La productividad se expresó como: i) eficiencia biológica (EB) (Tschierpe y Hartmann, 1977) y ii) tasa de producción (Salmones y Durán Barradas, 2001). Los hongos cosechados se agruparon por tamaño del píleo: (G1) hasta $5 \mathrm{~cm}$, (G2) de 5.1 a $10 \mathrm{~cm}$, (G3) 10.1 a 15 cm y grupo 4 (G4) >15 cm (Gaitán-Hernández y Salmones, 2008), al igual que la producción de basidiomas de cada cosecha expresado como porcentaje del volumen total de hongos obtenidos.

\section{RESULTADOS Y DISCUSIÓN}

\section{Obtención de micelios monocarióticos y cruzas}

En los procesos de hibridación y mejoramiento genético, la selección de parentales apropiados es un factor esencial. En el presente estudio se eligieron parentales de diferente origen geográfico con la finalidad de contar con mayor variabilidad genética de la especie, que evitara la repetición de alelos durante la recombinación genética.

Los 12 cultivos monospóricos de las tres cepas parentales se agruparon en cuatro tipos de compatibilidad, lo que concuerda con el patrón de sexualidad heterotálico tetrapolar de Pleurotus (Eger, 1978; Shnyreva y Shtaer, 2006). Cada tipo de compatibilidad fue conformado por uno a cuatro cultivos monospóricos. A los 10 d de incubación, los cultivos monospóricos de la cepa IE-717 presentaron diámetros de micelio de hasta 72.7 $\pm 2.3 \mathrm{~mm}$, mientras que los monospóricos de IE-115 crecieron entre $59.3 \pm 1.0$ hasta $64.8 \pm 2.5 \mathrm{~mm}$ y los cultivos de IE-739 de $53.5 \pm 1.6$ a $69.0 \pm 2.0$ mm (Tabla 
1). Los diámetros miceliales más desarrollados correspondieron a los monospóricos 4 y 5 del parental IE-717 y al monospórico 9 del parental IE-739, por lo que fueron estadísticamente similares entre ellos y diferentes al resto de micelios evaluados.

En promedio, los cultivos monospóricos requirieron de 14 a 20 d para cubrir el diámetro total de la caja de Petri (90 mm Ø). De acuerdo con los resultados del análisis estadístico aplicado a los diámetros desarrollados por los 36 micelios monospóricos a los 10 d de incubación, se eligió un cultivo de cada tipo de compatibilidad para las cruzas interespécimen. Como resultado del entrecruzamiento de los cuatro tipos de apareamiento de las tres cepas parentales se obtuvieron 48 micelios dicarióticos, a los cuáles se les asignó un código de identificación (Tabla 1). Estos cultivos cubrieron el diámetro total de la caja de Petri en 10-14 d de incubación. Los resultados de crecimiento micelial de parentales y cruzas a los $8 \mathrm{~d}$ de incubación se muestran en la Figura 1.

El germoplasma obtenido de la combinación de los parentales IE-115 e IE-717 alcanzó diámetros miceliales promedio de $51.0 \pm 0.9$ (C9) a $73.8 \pm 1.2 \mathrm{~mm}$ (C3), mientras que las combinaciones de IE-115 x IE-739 va- riaron entre $47.0 \pm 1.1$ (C31) hasta $68.1 \pm 1.9 \mathrm{~mm}$ (C17) y las de IE-717 x IE-739 desde $39.3 \pm 0.9$ (C39) hasta 70.2 $\pm 1.5 \mathrm{~mm}$ (C37). Las cepas parentales presentaron diámetros promedio de $64.5 \pm 3.2 \mathrm{~mm}$ (IE-115), $50.2 \pm 2.4$ $\mathrm{mm}$ (IE-717) y $60.1 \pm 4.72 \mathrm{~mm}$ (IE-739), siendo disímil entre algunas cepas y sus parentales $(p<0.05)$ (Figura 1). Once de los dicariones (C1-C7, C10, C16-C18) obtenidos a partir de IE-115 presentaron un crecimiento micelial mayor o similar a su parental, con diferencias significativas con algunos de los dicariones restantes. De manera similar, 11 dicariones (C17-C19, C21, C24, C26, C33, C34, C36, C37 y C48) de la progenie obtenida con IE-739 presentaron diámetros miceliales mayores a similares de su parental. IE-717 mostró menor desarrollo micelial que los otros parentales, por lo que gran parte de su progenie ( 25 cruzas) logró superar el tamaño del micelio desarrollado por su parental, de éstas 13 correspondieron a las combinaciones con IE115 y los 12 restantes con IE-739.

En general se observó que la combinación entre IE115 e IE-717 generó un mayor número de cruzas con mejor tasa de crecimiento micelial que sus parentales, que superaron los $70 \mathrm{~mm}$ de diámetro micelial a los

TABLA 1. Crecimiento micelial de los cultivos monospóricos de Pleurotus pulmonarius a los 10 días de incubación y origen de las cruzas evaluadas en el estudio

\begin{tabular}{|c|c|c|c|c|c|c|c|c|}
\hline $\begin{array}{c}\text { CEPA } \\
\text { PARENTAL }\end{array}$ & $\begin{array}{c}\text { TIPOS DE } \\
\text { COMPATIBILIDAD }\end{array}$ & $\begin{array}{c}\text { No. DE } \\
\text { MONOSPÓRICO }\end{array}$ & $\begin{array}{l}\varnothing \text { MICELIAL } \\
\text { (MM) }\end{array}$ & PARENTALES & & $\begin{array}{l}\text { CÓDIGO } \\
\text { COMBINACIÓN }\end{array}$ & $\begin{array}{l}\text { IGNADO Y } \\
\text { MONOSPÓRICOS }\end{array}$ & \\
\hline \multirow{4}{*}{$\mathrm{IE}-115$} & I (A1B1) & 3 & $59.8 \pm 1.6 c^{1}$ & \multirow{4}{*}{$\begin{array}{c}\text { IE-115 } \\
X \\
\text { IE-717 }\end{array}$} & $C 1=4-4$ & $C 2=11-4$ & $C 3=10-5$ & $C 4=10-11$ \\
\hline & II $\left(A_{1} B_{2}\right)$ & 4 & $64.8 \pm 2.5 b$ & & $C 5=4-5$ & $C 6=11-5$ & $C 7=10-4$ & $C 8=3-11$ \\
\hline & III $\left(A_{2} B_{1}\right)$ & 11 & $59.3 \pm 1.0 c$ & & $C 9=3-9$ & $\mathrm{C} 10=4-11$ & $\mathrm{C} 11=10-9$ & $C 12=11-9$ \\
\hline & II $\left(\mathrm{A}_{2} \mathrm{~B}_{2}\right)$ & 10 & $64.3 \pm 1.2 b$ & & $C 13=4-9$ & $\mathrm{C} 14=11-11$ & $C 15=3-5$ & $C 16=3-4$ \\
\hline \multirow{4}{*}{ IE-717 } & I $\left(A_{3} B_{3}\right)$ & 4 & $69.2 \pm 2.1 \mathrm{a}$ & \multirow{4}{*}{$\begin{array}{c}\mathrm{IE}-115 \\
\mathrm{X} \\
\mathrm{IE}-739\end{array}$} & $C 17=11-7$ & $C 18=3-7$ & $\mathrm{C} 19=4-7$ & $C 20=11-9$ \\
\hline & II $\left(A_{3} B_{4}\right)$ & 5 & $72.7 \pm 2.3 a$ & & $C 21=10-9$ & $C 22=4-9$ & $C 23=3-9$ & $C 24=3-4$ \\
\hline & III $\left(A_{4} B_{3}\right)$ & 9 & $57.3 \pm 0.5 \mathrm{~cd}$ & & $C 25=11-1$ & $C 26=10-7$ & $C 28=4-1$ & $C 28=3-1$ \\
\hline & II $\left(\mathrm{A}_{4} \mathrm{~B}_{4}\right)$ & 11 & $63.2 \pm 3.8 b$ & & $C 29=10-4$ & C30 = 10-1 & $C 31=4-4$ & $C 32=11-4$ \\
\hline \multirow{4}{*}{ IE-739 } & I $\left(A_{5} B_{5}\right)$ & 1 & $59.3 \pm 1.2 c$ & \multirow{4}{*}{$\begin{array}{c}\text { IE-717 } \\
X \\
\text { IE-739 }\end{array}$} & $\mathrm{C} 33=5-9$ & $C 34=5-7$ & $C 35=4-4$ & $C 36=9-9$ \\
\hline & II $\left(A_{5} B_{6}\right)$ & 4 & $53.5 \pm 1.6 \mathrm{e}$ & & $\mathrm{C} 37=4-7$ & $\mathrm{C} 38=9-1$ & $C 39=11-1$ & $C 40=5-1$ \\
\hline & III $\left(A_{6} B_{5}\right)$ & 9 & $69.0 \pm 2.0 a$ & & $\mathrm{C} 41=11-4$ & $C 42=4-1$ & $C 43=11-9$ & $C 44=9-7$ \\
\hline & II $\left(A_{6} B_{6}\right)$ & 7 & $54.8 \pm 0.8 \mathrm{de}$ & & $C 45=4-9$ & $C 46=5-4$ & $C 47=11-7$ & $C 48=9-4$ \\
\hline
\end{tabular}

${ }^{1}$ Diámetro micelial desarrollado a los 10 días de incubación. Letras diferentes en la misma columna, para todas las cepas, indican diferencias significativas $(a=0.05)$, de acuerdo a la prueba de intervalos múltiples de Tukey. 
IE-115 x IE-717
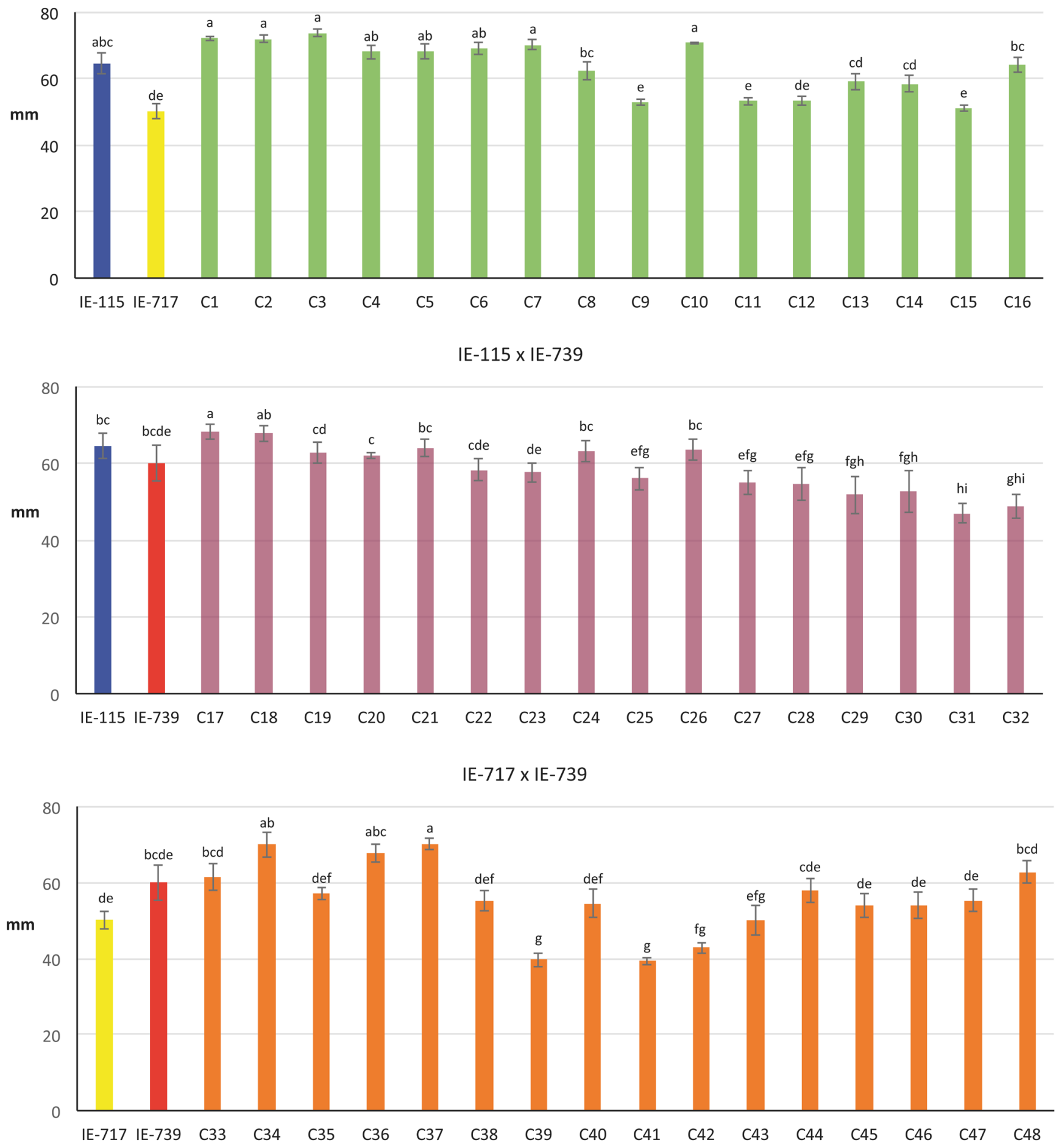

FiguRa 1. Diámetro micelial promedio $(\mathrm{mm})$ de los parentales y cruzas obtenidas a los 10 días de incubación. En cada combinación de parentales, columnas que no comparten al menos una letra en común, indican diferencias estadísticas, de acuerdo con la prueba de intervalos múltiples de Tukey $(p=0.05)$. 
8 d de incubación. Si bien se ha reportado que la capacidad de una cepa para colonizar de manera rápida y eficiente el sustrato en que crece es una característica independiente de su capacidad para la formación y desarrollo de fructificaciones (Royse, 2002; Gaitán-Hernández y Salmones, 2008), es recomendable para fines de cultivo comercial cultivar germoplasma con rápido crecimiento micelial, porque provee ventajas sobre la expresión de enzimas lignocelulolíticas como lacasas, relacionadas no sólo con el proceso de colonización y descomposición del sustrato, sino también con la capacidad de defensa ante organismos contaminantes y consecuentemente, en la disponibilidad de nutrientes requeridos para la formación y desarrollo de fructificaciones (Mata et al., 2005, 2017).

El crecimiento rápido de basidiomicetos se debe a factores poligénicos, predominantemente controlado por uno o dos genes, demostrado a través de la identificación del loci de caracteres cuantitativos (QTL, Quantitative Trait Loci) en P. ostreatus (Larraya et al., 2002). En este estudio es interesante destacar que la mayoría de la progenie del monospórico 7 y 4 del parental IE-739 e IE-115 respectivamente, mantuvieron un rápido crecimiento micelial y altos valores de productividad en los dicariones obtenidos. Lo anterior podría deberse a la presencia de los genes involucrados en el proceso. En $P$. ostreatus se ha observado que los monocariones de rápido crecimiento pertenecen a tipos de compatibilidad específicos (Larraya et al., 2002). Una posible explicación a la asociación entre el crecimiento micelial y los loci de reconocimiento sería que las fuerzas evolutivas de estos sistemas difieren del resto del genoma, ya que la selección equilibrada y la recombinación suprimida parecen jugar un papel importante en su evolución (van der Nest et al., 2009).

\section{Producción de las fructificaciones}

En las 48 cruzas obtenidas, el micelio invadió adecuadamente el sustrato, con dos a tres semanas de incubación para cubrirlo totalmente. Sin embargo, dos dicariones no lograron presentar primordios, por lo que los resultados de productividad corresponden a las 46 cepas restantes. En gran parte de las muestras cultivadas se observó que el micelio desarrollado sobre la paja mostraba zonas más densas con aspecto aterciopelado, que con el paso de los días se convirtió en una cubierta tipo costra, aunque sin alcanzar la compactación observada en otras especies cultivadas como Len- tinula edodes (Berk.) Pegler. El desarrollo cespitoso de los primordios y la morfología de los basidiomas cosechados fue característico de la especie, flabeliformes e infundibuliformes, de color blanquecino a crema, algunos con tonalidades marrón-grisáceas.

En la Figura 2A y B se presenta la distribución de las cruzas de acuerdo con los valores de EB y TP registrados. La eficiencia biológica de las cepas progenie fluctuó entre 16.4 (C36) hasta $147.5 \%$ (C37) y la TP entre 0.14 (C43) a $2.59 \%$ (C35). Del total de cruzas, 12, 21, y 13 alcanzaron EBs $<50,50-80$ y $>80 \%$ respectivamente. Las cepas parentales presentaron EBs desde 91.9 (IE-739) hasta $114.8 \%$ (IE-115). En cuanto a las TPs, 17, 13, 12 y 4 cruzas presentaron $<1,1-1.5,1.5-2$ y $>2 \%$ respectivamente; mientras que en las cepas parentales los porcentajes fluctuaron entre 1.45 (IE-739) a 2.07\% (IE-115). Las cepas produjeron entre 1 a 4 cosechas durante ciclos de cultivo que fluctuaron entre 40 y $98 \mathrm{~d}$. El patrón de distribución de hongos obtenidos por cosecha fue variable, aunque en general la mayor cantidad se desarrolló en las primeras dos cosechas, con un porcentaje acumulado de $<70$ a $99 \%$ de la producción total. En la Figura $2 \mathrm{C}$ se muestra la distribución de las cruzas de acuerdo con los porcentajes alcanzados. En cuanto a las cepas parentales, estos porcentajes correspondieron al 76.7 (IE-717), 79.4 (IE-739) y 96.9 \% (IE-115). Esta característica es altamente deseable en la producción comercial, ya que representa la factibilidad de lograr un mayor número de ciclos de cultivo durante el año.

Con respecto al tamaño de los hongos, se desarrollaron especímenes de los 4 grupos considerados, correspondiendo a los grupos 1 y 2 los mayores porcentajes (Figura 2D); mientras que en las cepas parentales los valores promedio de los grupos fueron de 62.5 (G1), 22.6 (G2), 9.9 (G3) y 5 \% (G4).

En la Tabla 2 se presentan los datos de productividad de las 13 cepas progenie que registraron EBs $>80 \%$, así como de sus cepas parentales. Dos de las cepas provienen del entrecruzamiento entre los parentales IE-115 e IE-717 (C1 y C2), cuatro de la combinación entre IE-115 y IE-739 (C17-C19 y C21); y las 6 cepas restantes del entrecruzamiento entre IE 717 y IE-739 (Tabla 1). La mayoría de las cruzas presentaron valores de productividad superiores al menos de uno de sus parentales, con excepción de la cruza C37 que alcanzó una EB de $147.5 \%$, el valor promedio más alto en este estudio. Las cruzas C34 y C35 también resultaron altamente productivas, con $139 \%$ de EBs. 

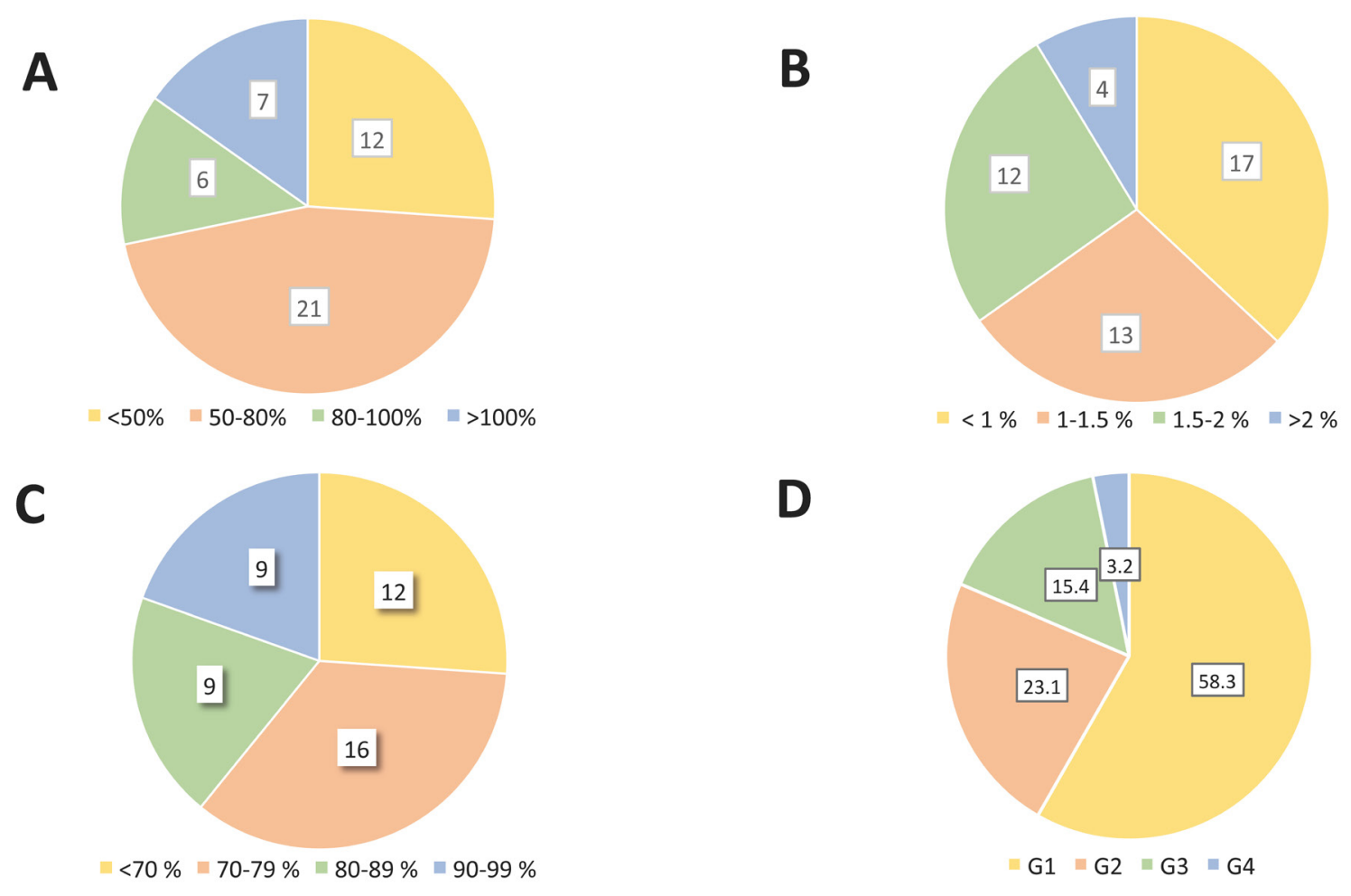

FIGURA 2. Características de producción de las 46 cepas progenie cultivadas en paja de cebada. A: eficiencia biológica, B: tasa de producción, C: relación de las primeras dos cosechas con respecto al total de la producción obtenida y D: Tamaño de los basidiomas desarrollados. Los valores corresponden a la sumatoria (A-C) y porcentaje promedio (D) de todas las cepas.

TABLA 2. Productividad de las cepas parentales y las cruzas que alcanzaron las eficiencias biológicas más altas en el estudio (>80 \%)

\begin{tabular}{|c|c|c|c|c|c|c|}
\hline CEPA & PRIMORDIOS (DÍAS) & 1A. COSECHA (DÍAS) & Cosechas & $\begin{array}{c}\text { CicLo dE CULTIVO } \\
\text { (DÍAS) }\end{array}$ & $\begin{array}{l}\text { EB } \\
\%\end{array}$ & $\begin{array}{l}\mathrm{TP} \\
\%\end{array}$ \\
\hline IE-115 & $17-20^{1}$ & $21-26$ & $3-4$ & $48-62$ & $114.8 a-d^{2}$ & $2.07 a-d$ \\
\hline IE-717 & $24-26$ & $28-32$ & $1-4$ & $40-68$ & $102.9 \mathrm{bcd}$ & $1.97 \mathrm{~b}-\mathrm{e}$ \\
\hline IE-739 & $26-29$ & $33-35$ & $3-4$ & $55-68$ & $91.9 d$ & $1.65 c-f$ \\
\hline $\mathrm{C} 1$ & $26-28$ & $32-34$ & $2-4$ & $49-69$ & $98 \mathrm{~cd}$ & $1.51 \mathrm{def}$ \\
\hline C2 & $23-27$ & $30-34$ & $3-4$ & $54-69$ & 117.7a-d & $1.81 \mathrm{cde}$ \\
\hline C17 & $25-26$ & $30-32$ & $3-4$ & $50-66$ & $92.7 d$ & $1.50 \mathrm{def}$ \\
\hline $\mathrm{C} 18$ & $23-28$ & $30-34$ & $3-4$ & $58-66$ & $100.3 \mathrm{~cd}$ & $1.56 \mathrm{def}$ \\
\hline C19 & $20-22$ & $24-28$ & $3-4$ & $64-93$ & $96.5 \mathrm{bcd}$ & $1.40 \mathrm{ef}$ \\
\hline $\mathrm{C} 21$ & $20-21$ & $26-27$ & $3-4$ & $61-83$ & $131.4 \mathrm{abc}$ & 2.03a-d \\
\hline $\mathrm{C} 22$ & $25-28$ & $33-34$ & $1-4$ & $43-70$ & $81.4 d$ & $1.55 \mathrm{def}$ \\
\hline C34 & $28-37$ & $35-44$ & $1-4$ & $44-62$ & 139.6ab & $2.46 a b$ \\
\hline C35 & $24-30$ & $30-35$ & $3-4$ & $49-51$ & $139.5 \mathrm{ab}$ & $2.59 a$ \\
\hline C37 & $38-39$ & $42-43$ & $2-4$ & $57-76$ & 147.5 a & 2.19abc \\
\hline $\mathrm{C} 40$ & $41-56$ & $47-66$ & 3 & $63-81$ & $82.9 d$ & $1.12 f$ \\
\hline C42 & $30-32$ & $35-37$ & $3-4$ & $56-79$ & $85.8 \mathrm{~d}$ & $1.15 f$ \\
\hline $\mathrm{C} 46$ & $41-47$ & $47-54$ & 3 & $63-75$ & $83.8 d$ & $1.13 f$ \\
\hline
\end{tabular}

${ }^{2}$ Resultados en una columna con al menos una misma letra indica que no hubo diferencias significativas entre los valores, de acuerdo con la prueba de intervalos múltiples de Tukey $(p=0.05)$. 
Los tiempos requeridos por los parentales para presentar los primordios variaron entre 17 (IE-115) hasta 26 d (IE-739) y la primera cosecha a partir del día 21 (IE-115) y hasta el día 33 (IE-739). Se evaluó entre 1 (IE-717) a 4 cosechas en ciclos de cultivo de 40 hasta $68 \mathrm{~d}$ (Tabla 2). En cuanto a las cepas progenie, los primeros primordios se observaron a partir del día 20 (C19 y C20) y hasta el día 41 (C40 y C46). En las cepas progenie se evaluaron de 1 a 4 cosechas, en ciclos de cultivo entre 43 y 93 d (Tabla 2). Aunque existió una variabilidad alta en los parámetros de cultivo evaluados, los resultados son similares y/o superiores a los reportados en trabajos previos de P. pulmonarius cultivado en diferentes sustratos (Salmones y Durán Barradas,
2001; Philippoussis et al., 2001; Gaitán-Hernández et al., 2009; Nyochembeng et al., 2008; Gupta et al., 2011; Adebayo et al., 2013; Abdulgani et al., 2017).

La mayoría de los especímenes cosechados correspondieron por su tamaño al grupo G1, con excepción de las cruzas C40 y C46 que presentaron una mayor producción de fructificaciones de G2 y G3 (Figura 3). Entre los parentales se distinguió el porcentaje de basidiomas desarrollados por IE-717 e IE-739, que correspondieron al 13.5 y $15.5 \%$ (G3), y 9.8 y $5.1 \%$ (G4) respectivamente de la producción total.

En cuanto a la progenie, las dos cepas resultantes de las combinaciones entre IE-115 e IE-717 no mostraron incremento en el tamaño de las fructificaciones con
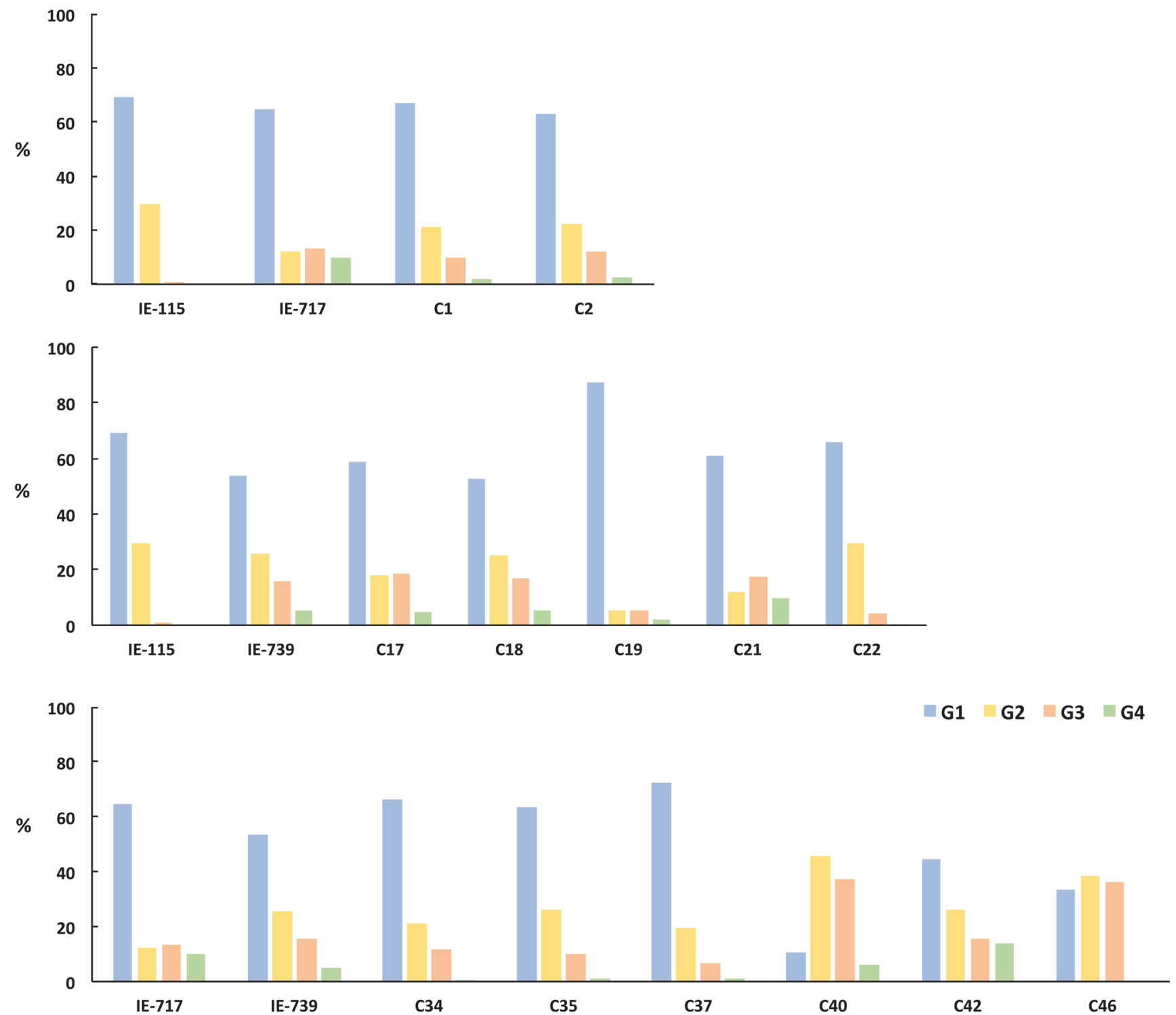

FIGURA 3. Porcentaje de basidiomas desarrollados por las cepas parentales y sus cruzas cultivadas en paja de cebada, agrupadas de acuerdo con el tamaño del píleo, (G1) hasta $5 \mathrm{~cm}$, (G2) de 5.1 a $10 \mathrm{~cm}$, (G3) 10.1 a $15 \mathrm{~cm}$ y grupo 4 (G4) <15 cm. 
relación a sus parentales, ya que mantuvieron un alto porcentaje de G1 e incluso menor del G4 que IE-717. En cuanto a la descendencia de IE-115 e IE-739, destaca la producción de las cruzas C17, C18 y C21 que, con porcentajes promedio de $18.4,17.1$ y $17.2 \%$ para el grupo 3, superaron los valores de sus parentales de 0.9 \% (IE-115) y $15.5 \%$ (IE-739). En cuanto a G4, sólo las cepas C18 (5.4\%), C21 (9.7\%) e IE-739 (5.1\%) lograron desarrollar basidiomas con píleos mayores a 15 $\mathrm{cm}$ de diámetro.

Con respecto a las combinaciones de la progenie de IE-717 e IE-739, destacan las cepas C40, C42 y C46 que presentaron un equilibrio entre los porcentajes de los grupos, especialmente la C42 logró un alto porcen- taje de G4, se asume heredada de su parental IE-717. Con excepción de IE-115 que no produjo fructificaciones con píleo mayor a $15 \mathrm{~cm}$ de $\varnothing$, las otras dos cepas parentales fueron altamente productivas con basidiomas G3 y G4, que correspondieron al 23.3 (IE-717) y $20.6 \%$ (IE-739) de la producción total. Estos valores son equiparables (Salmones y Durán Barradas, 2001) y superiores a estudios previos (Gaitán-Hernández et al., 2009).

En la Figura 4 se observa la distribución de las fructificaciones durante las cosechas, una característica de interés para los productores porque está relacionada con la factibilidad de incrementar el número de ciclos de cultivo por área de producción. Para las cruzas C1 y C2, las
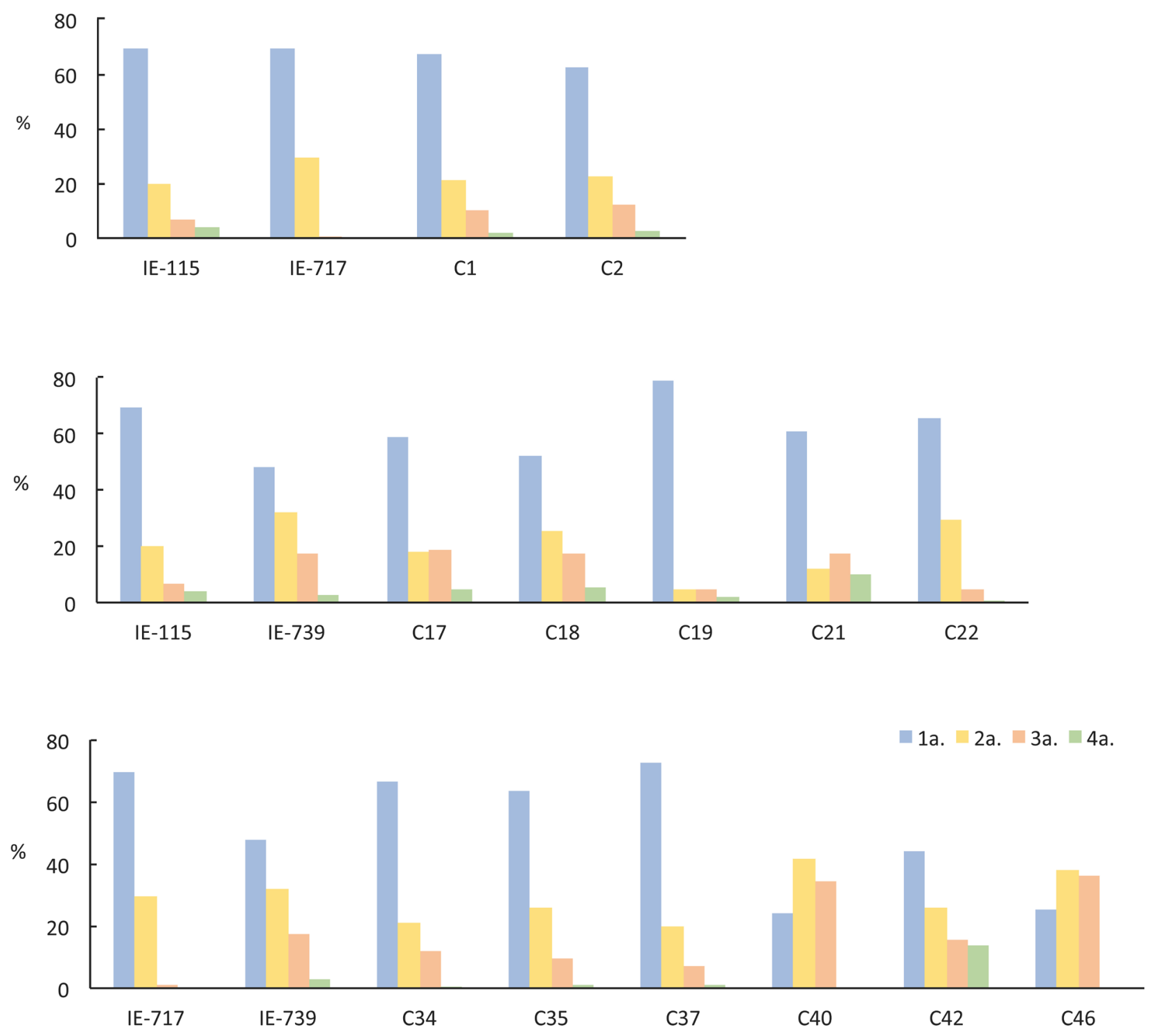

FIGURA 4. Distribución de la producción de basidiomas desarrollados por las cepas parentales y sus cruzas en las diferentes cosechas evaluadas. 
primeras dos cosechas correspondieron al 88.1 y 85.1 $\%$ de la producción total, valor similar al $89.2 \%$ de su parental IE-115, aunque más bajo al $99.2 \%$ registrado por IE-717. En cuanto a la progenie de la combinación IE-115 × IE-739, sólo las cruzas C19 (83.6\%) y C22 (95.2 \%) lograron porcentajes mayores al promedio de 79.9 \% registrado por IE-739, aunque ninguna cruza superó el $89.2 \%$ del parental IE-115. De las cruzas resultantes de IE-717 × IE-739, sólo tres cepas, C34 (87.5\%), C35 (89.3 \%) y C37 (92.1\%), superaron los porcentajes de uno de sus parentales (IE-739), sin que ninguna lograra superar el $99.2 \%$ registrado por IE-717.

Es importante destacar que las condiciones ambientales pudieron influir en el desarrollo y productividad de las cepas. Por el número de cruzas obtenidas y las limitaciones de espacio para la experimentación, el proceso de cultivo duró aproximadamente dos años, durante este periodo se trató de mantener estable la temperatura ambiental en el área de producción, lo que resultó complicado especialmente durante los meses calurosos, parámetro que influyó negativamente en el comportamiento de las cepas, al retrasar la inducción de los primordios y consecuentemente, en los ciclos de cultivo.

En cuanto a las características productivas de las cepas progenie y a pesar de lo azaroso del proceso, se lograron resultados satisfactorios, ya que el $25 \%$ del germoplasma obtenido alcanzó altos valores de eficiencia biológica y tasa de producción, que superó el $100 \%$ de EB y el $2 \%$ de TP, características de interés para el sector productivo, ya que representa incrementar sus ingresos al tener la factibilidad de aumentar el número de ciclos de cultivo en una misma área de producción.

\section{AGRADECIMIENTOS}

Los autores agradecen al Consejo Nacional de Ciencia y Tecnología (CONACYT) el financiamiento otorgado a través del Proyecto FORDECYT-273647, así como a las autoridades del Instituto de Ecología A. C. las facilidades otorgadas para realizar el estudio. Se agradece también el apoyo técnico otorgado por la M. en C. Rosalía Pérez y la Dra. Dulce Ma. Murrieta en el desarrollo experimental de este trabajo.

\section{LITERATURA CITADA}

Abdulgani, R., C.C. Lau, N. Abdullah, W. Vikineswary, 2017. Morphological and molecular characterization of Pleurotus pulmonarius hybrids with improved sporophore features and higher biological efficacy. International Journal of Agriculture and Biology 19: 707-712.
Adebayo, E.A., J.K. Oloke, A. Yadav, M. Barooah, T.C. Bora, 2013. Improving yield performance of Pleurotus pulmonarius through hyphal anastomosis fusion of dikaryons. World Journal of Microbiology and Biotechnology 29: 1029-1037.

Avin, F.A., S. Bhassu, V. Rameeh, Y.S. Tan, S. Vikineswary, 2016. Genetics and hybrid breeding of Pleurotus pulmonarius: heterosis, heritability and combining ability. Euphytica 209: 85-102.

Chang, S.T., P.G. Miles, 2004. Mushrooms: Cultivation, nutritional value, medicinal effect, and environmental impact. 2 ed. CRC Press, Boca Raton.

Eger, G., 1978. Biology and breeding of Pleurotus. In: Chang, S.T., W.A. Hayes (eds.), The biology and cultivation of edible mushrooms. Academic Press, Nueva York. Pp. 497-519.

Gaitán-Hernández, R., D. Salmones, 2008. Obtaining and characterizing Pleurotus ostreatus strains for commercial cultivation under warm environmental conditions. Scientia Horticulturae 118: 106-110.

Gaitán-Hernández, R., D. Salmones, R. Pérez Merlo, G. Mata, 2009. Evaluación de la eficiencia biológica de cepas de Pleurotus pulmonarius en paja de cebada fermentada. Revista Mexicana de Micología 30: 63-71.

Gupta, B., B.P. Niranjan Reddy, A.S. Kotasthane, 2011. Molecular characterization and mating type analysis of oyster mushroom (Pleurotus spp.) using single basidiospores for strain improvement. World Journal Microbiology and Biotechnology 27: 1-9.

Guzmán, G., 2000. Genus Pleurotus (Jacq.: Fr.) P. Kumm. (Agaricomycetideae): diversity, taxonomic problems, and cultural and traditional medicinal uses. International Journal of Medicinal Mushrooms 2: 95-123.

Guzmán, G., G. Mata, D. Salmones, C. Soto-Velazco, L. Guzmán-Dávalos, 2013. El cultivo de los hongos comestibles, con especial atención a especies tropicales y subtropicales en esquilmos y residuos agroindustriales. 3a Reimpresión. Instituto Politécnico Nacional, México, D.F.

Larraya, L.M., E. Idareta, D. Arana, E. Ritter, A.G. Pisabarro, L. Ramírez, 2002. Quantitative trait loci controlling vegetative growth rate in the edible basidiomycete Pleurotus ostreatus. Applied and Environmental Microbiology 68: 1109-1114.

Lechner B.E., J.E. Wright, E. Albertó, 2005. The genus Pleurotus in Argentina: mating tests. Sydowia 57: 233-245.

Martínez-Carrera, D., A. Larqué-Saavedra, A. Tovar Palacio, N. Torres, M.E. Meneses, M. Sobal Cruz, P. Morales Almora, M. Bonilla Quintero, H. Escudero Uribe, I. Tello Salgado, T. Bernabé-González, W. Martínez Sánchez, Y. Mayett, 2016. Contribución de los hongos comestibles, funcionales y medicinales a la construcción de un paradigma sobre la producción, la dieta, la salud y la cultura en el sistema agroalimentario de México. In: Ciencia, tecnología e innovación en el sistema agroalimentario mexicano. Martínez-Carrera, D., J. Ramírez Juárez (eds.). COLPOS-AMC-CONACYT-UPAEP-IMINAP, Texcoco. Pp. 581-640.

Mata, G., R. Gaitán-Hernández, D. Salmones, 2013. Biotechnology for edible mushroom culture: a tool for sustainable development in Mexico. In: Yañez-Arancibia, A., R. Dávalos Sotelo, J.W. Day, E. Reyes (eds.), Ecological dimensions for sustainable socioeconomic development. WIT Press, Southampton. Pp. 483-506. 
Mata, G., D.M. Murrieta Hernández, L.G. Iglesias Andreu, 2005. Changes in lignocellulolytic enzyme activites in six Pleurotus spp. strains cultivated on coffee pulp in confrontation with Trichoderma spp. World Journal of Microbiology and Biotechnology 21: 143-150.

Mata, G., D. Salmones, J.M. Savoie, 2017. Las enzimas ligninocelulolíticas de Pleurotus spp. In: Sánchez, J.E., D. Royse (eds.), La biología, el cultivo, las propiedades nutricionales y medicinales de las setas Pleurotus spp. El Colegio de la Frontera Sur, San Cristobal de las Casas. Pp. 63-82.

Nyochembeng, L.M., C.A. Beyl, R.P. Pacumbaba, 2008. Optimizing edible fungal growth and biodegradation of inedible crop residues using various cropping methods. Bioresource Technology 99: 5645-5649.

Philippoussis, A., G. Zervakis, P. Diamantopoulou, 2001. Bioconversion of agricultural lignocellulosic wastes through the cultivation of the edible mushrooms Agrocybe aegerita, Volvariella volvacea and Pleurotus spp. World Journal of Microbiology and Biotechnology 17: 191-200.

Pánek, M., L. Wiesnerová, I. Jablonský, D. Novotný, M. Tomsovsky, 2019. What is cultivated oyster mushrooms? Phylogenetic and physiological study of Pleurotus ostreatus and related species. Mycological Progress 18: 1173-1186.
Royse, D.J., 2002. Influence of spawn rate and commercial delayed release nutrient levels on Pleurotus cornucopiae (oyster mushroom) yield, size, and time to production. Applied Microbiology and Biotechnology 58: 527-531.

Salmones, D., Z. Durán Barradas, 2001. Obtaining and selecting highly productive strains of Pleurotus pulmonarius under warm environmental conditions. Mushroom Research 10: 59-65.

Shnyreva, A.A., O.V Shtaer, 2006. Differentiation of closely related oyster fungi Pleurotus pulmonarius and $P$. ostreatus by mating and molecular markers. Russian Journal of Genetics 42: 539-545.

StatSoft Inc., 2005. STATISTICA para Windows versión 7.1. www.statsoft.com

Tschierpe, H.J., K.H. Hartmann, 1977. A comparison of different growing methods. Mushroom Journal 60: 404-416.

van der Nest, M.A., B. Slippers, E.T. Steenkam, L. de Vos, K. Van Zyl, J. Stenlid, M.J. Wingfield, B.D. Wingfield, 2009. Genetic linkage map of Amylostereum aerolatum reveals an association between vegetative growth and sexual and self-recognization. Fungal Genetics and Biology 46: 632-641. 